\begin{tabular}{|c|c|}
\hline Title & Investigation of Current Flow Between Turns of NI REBCO Pancake Coil by 2-D Finite-Element Method \\
\hline Author(s) & Noguchi, So; Monma, Katsutoshi; Igarashi, Hajime; Ishiyama, A tsushi \\
\hline Citation & $\begin{array}{l}\text { IEEE Transactions on A pplied Superconductivity, 26(3), } 4901205 \\
\text { https://doi.org/10.1109/T A SC.2016.2536945 }\end{array}$ \\
\hline Issue Date & 2016-04 \\
\hline Doc URL & http:/hdl.handle.net/2115/62181 \\
\hline Rights & $\begin{array}{l}\text { C } 2016 \text { IEEE. Personal use of this material is permitted. Permission from IEEE must be obtained for all other uses, in } \\
\text { any current or future media, including reprinting/republishing this material for advertising or promotional purposes, } \\
\text { creating new collective works, for resale or redistribution to servers or lists, or reuse of any copyrighted component of } \\
\text { this work in other works. }\end{array}$ \\
\hline Tyре & article (author version) \\
\hline File Information & EUCAS_fullpaper_after_minor_revision.pdf \\
\hline
\end{tabular}

Instructions for use 


\title{
Investigation of Current Flow between Turns of NI REBCO Pancake Coil by 2-D Finite Element Method
}

\author{
So Noguchi, Katsutoshi Monma, Hajime Igarashi, and Atsushi Ishiyama
}

\begin{abstract}
The No-Insulation (NI) winding technique for NI REBCO pancake coil is expected to improve the dynamic and thermal stability and to enhance the current density. The investigations of the electromagnetic and thermal behaviors are important for the development of NI REBCO coils. Many stability investigations of the NI REBCO coil itself have been carried out by experiments and simulations. However, the detailed behavior of the bypass current between turns has been not shown yet. Although the contact resistivity was obtained through the priori experiment, it included the resistivity of not only contact surface but also the components, i.e., the copper stabilizer and the Hastelloy substrate, of REBCO tape. To investigate the detailed bypass current behavior in this paper, the true contact surface resistivity is taken into account in the simulation. The bypass current on the cross section of NI REBCO tape is simulated using the 2-D FEM. From the simulation results, the influence of the coil-radial resistivity between turns on the turn-to-turn contact surface resistivity is clarified. In addition, the heat loss is also reported, and a simple equivalent circuit of the turn-to-turn contact is proposed.
\end{abstract}

Index Terms-Electromagnetic simulation, NI REBCO pancake coil, No-Insulation winding technique, turn-to-turn contact surface resistivity.

\section{INTRODUCTION}

$\mathrm{R}$ ECENTRY, the No-Insulation (NI) winding technique [1], [2] has received attention. This technique can be applied to REBCO pancake coils for the use of NMR [3], MRI, and accelerator applications. The NI winding technique enhances stability and current density simultaneously; when a local hotspot appears in an NI REBCO coil, the transport current can avoid it and flows directly into the adjacent turns. This behavior is expected to improve the thermal stability. Additionally, owing to no insulation material, the current density can be increased. However, the drawback to the NI winding technique is the charging delay [4]. So far, to confirm the effectiveness of the NI winding technique, some current flow simulations in a pancake coil have been performed using the simple equivalent electrical circuit model [5], [6] or the partial element equivalent circuit (PEEC) model [7]. Through these simulations [4]-[6] and experiments [1]-[3],[7] it was

Automatically generated dates of receipt and acceptance will be placed here; authors do not produce these dates. This work was supported in part by the Grant-in-Aid for Scientific Research (A) (Grant Number 26249036). Corresponding author: So Noguchi.

S. Noguchi, K. Monma, and H. Igarashi are with Graduate School of Information Science and Technology, Hokkaido University, Sapporo 0600814, Japan (e-mail: noguchi@ssi.ist.hokudai.ac.jp).

A. Ishiyama is with the Department of Electrical Engineering and Bioscience, Waseda University, Tokyo 169-8555, Japan confirmed the stability was sufficiently high as a whole NI REBCO pancake coil. However, for more effective use of NI technique, we have to investigate the contact resistivity, the stabilizer thickness, the substrate material, and so on [7].

Before the above-mentioned investigation, the detailed behavior of the bypass current between turns has to be clarified. Although a turn-to-turn constant resistivity of 70 $\mu \Omega \cdot \mathrm{cm}^{2}$ was obtained in [8], it is unknown how the bypass current flows from a turn to the adjacent turns. The clarification of the local turn-to-turn bypass current behavior develops the NI technique more; e.g., it is useful to determine the cross-sectional structure of the REBCO wire, such as the stabilizer thickness. To reveal the turn-to-turn bypass current behavior, in this paper, the 2-D FEM is carried out on the cross section of the NI REBCO tapes touching side by side with arbitrary contact conductivity. From the simulation results, we investigate the current behavior, the Joule heat, and the total turn-to-turn contact resistance, changing the true turnto-turn contact resistivity. Finally, we propose a simple equivalent circuit between REBCO layers, and it is useful to determine the parameters of REBCO tape structure, e.g., the stabilizer thickness.

\section{Simulation Model AND Method}

\section{A. Simulation Model}

Fig. 1(a) shows the schematic view of an NI REBCO pancake coil wound with a REBCO tape (SuperPower 2G HTS tape [9]) using the NI technique [1], [2]. A turn in the NI REBCO pancake coil electrically contacts to the adjacent turns with contact resistivity $\rho_{\mathrm{ct}}$. The REBCO tape consists of a superconductor layer, a Hastelloy substrate, a copper stabilizer, a silver overlayer, and buffer stacks. Fig. 1(b) shows the

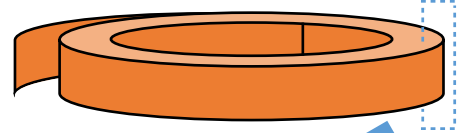

(a)

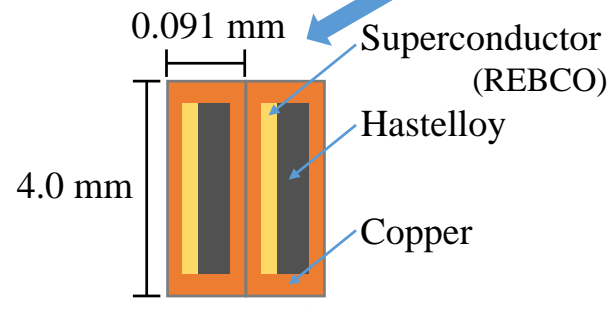

(b)

Fig. 1. (a) Schematic view of NI REBCO pancake coil. (b) Schematic view of simulation model aligning side by side with contact resistivity (not to scale). 
simulation model, where the silver overlayer is omitted and the buffer stacks are regarded as insulated because their influence is not so large in the current flow. The simulation model is the two cross sections of REBCO tape aligning side by side with arbitrary contact resistivity. Table I shows the parameters of the simulation model. There is no insulation material surrounding the REBCO tape surface, so that the current can flow from a turn to the adjacent tapes if a local normal-state transition occurs in the NI REBCO pancake coil.

\section{B. Simulation Method}

In the numerical simulation, the current flow between two adjacent turns is analyzed using the 2-D FEM. The governing equation in the simulation is given by

$\nabla \cdot \sigma \nabla \varphi=0$

where $\sigma$ and $\varphi$ are the electrical conductivity and the scalar potential, respectively. On the contact surface of the NI REBCO tapes, the double-nodes method [10] is employed. The electrical contact condition is as follows:

$\varphi_{l}-\varphi_{\mathrm{r}}=\rho_{\mathrm{ct}} J_{\mathrm{ct}}$

where $\varphi_{\mathrm{l}}, \varphi_{\mathrm{r}}, \rho_{\mathrm{ct}}$, and $J_{\mathrm{ct}}$ are the scalar potential on the contact surface of turns $l$ (the left cross section in Fig. 1 (b)) and $r$ (the right), the turn-to-turn contact surface resistivity, and the current density from the turn $l$ to $r$, respectively. In the simulation, the current per unit length of longitudinal direction from the left to the right REBCO layer in Fig. 1(b) is set to 1.0 $\mathrm{A} / \mathrm{cm}$. With these conditions, the current flow on the cross section of the NI REBCO tapes is simulated.

\section{SimULATION RESULTS}

\section{A. Current Density Distribution}

To investigate the influence of the turn-to-turn contact surface resistivity on the bypass current behavior, the simulation is performed on various turn-to-turn contact surface resistivities, $\rho_{\mathrm{ct}}=10^{-6}$ to $10^{4} \mu \Omega \cdot \mathrm{cm}^{2}$. Fig. 2(a) shows the simulated current density distributions when $\rho_{\mathrm{ct}}$ is $10^{-1}$ $\mu \Omega \cdot \mathrm{cm}^{2}$. In the simulation results, since the current flow on the top and bottom copper stabilizers of the NI REBCO tapes are symmetrical, only the enlarged top cross sections are presented. In Fig. 2(b), the current from the left to the right REBCO layer concentrates on the top copper stabilizer of the REBCO tape at $\rho_{\mathrm{ct}}=10^{-4} \mu \Omega \cdot \mathrm{cm}^{2}$. As shown in Fig. 2(c), when $\rho_{\mathrm{ct}}=10^{-1} \mu \Omega \cdot \mathrm{cm}^{2}$, the current flows along to the contact

TABLE I

PARAMETERS OF REBCO TAPE

\begin{tabular}{lcc}
\multicolumn{3}{c}{ PARAMETERS OF REBCO TAPE } \\
\hline \hline \multicolumn{1}{c}{ Parameter } & Unit & Value \\
\hline Tape width & {$[\mathrm{mm}]$} & 4.0 \\
Copper stabilizer thickness & {$[\mu \mathrm{m}]$} & 20 \\
REBCO thickness & {$[\mu \mathrm{m}]$} & 1.0 \\
REBCO width & {$[\mathrm{mm}]$} & 3.96 \\
Hastelloy thickness & {$[\mu \mathrm{m}]$} & 50 \\
Copper resistivity @ 77K & {$[\mu \Omega \cdot \mathrm{cm}]$} & 0.2 \\
Hastelloy resistivity [5] & {$[\mu \Omega \cdot \mathrm{cm}]$} & 125 \\
\hline \hline
\end{tabular}

surface, because the current cannot flow into the adjacent turn with a short length on the contact surface. When $\rho_{\mathrm{ct}}=10^{2}$ $\mu \Omega \cdot \mathrm{cm}^{2}$ in Fig. 2(d), the current passing through the contact surface uniformly flows into the adjacent turn.

Fig.3 shows the normalized current density on the turn-toturn contact surface. When $\rho_{\mathrm{ct}}=10^{-4} \mu \Omega \cdot \mathrm{cm}^{2}$, the length of current flowing is very short. The larger $\rho_{\mathrm{ct}}$, the longer the length of current passing through. When $\rho_{\mathrm{ct}}=10^{2} \mu \Omega \cdot \mathrm{cm}^{2}$, the current uniformly passes through the turn-to-turn contact surface.

\section{B. Coil-Radial Contact Resistivity}

The reference [4] showed the turn-to-turn contact resistivity, $70 \mu \Omega \cdot \mathrm{cm}^{2}$. However, its value includes the copper stabilizer and Hastelloy substrate resistivities, so it is termed "coil-radial contact resistivity" in this paper. The coil-radial contact resistivity $\rho_{\mathrm{r}}$ means the net resistivity from the left to the right

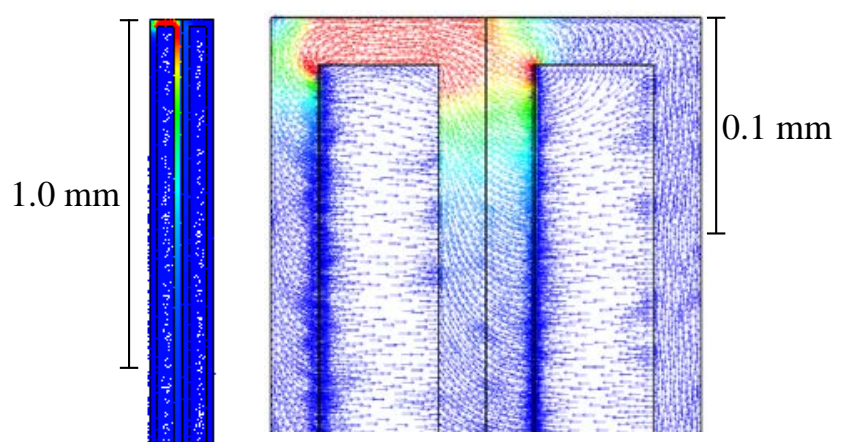

(b)

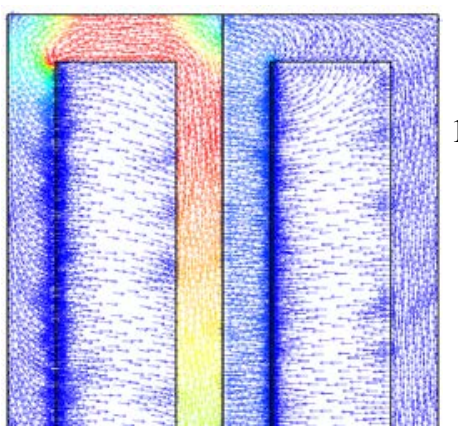

$\mathrm{A} / \mathrm{cm}^{2}$
100.0
80.0
60.0
40.0
20.0
0.0

(c)

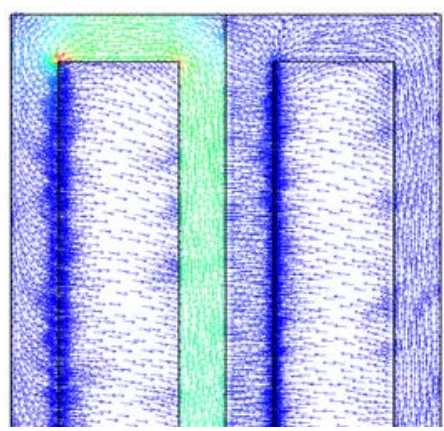

(d)

Fig. 2. Current density distribution on two cross sections of NI REBCO tapes at $\rho_{\mathrm{ct}}=$ (a) $10^{-1} \mu \Omega \cdot \mathrm{cm}^{2}$. (b)-(d) current density distributions on the top of cross section in NI REBCO tape when $\rho_{\mathrm{ct}}=$ (b) $10^{-4}$, (c) $10^{-1}$, and (d) $10^{2}$ $\mu \Omega \cdot \mathrm{cm}^{2}$, respectively. 
REBCO layer in Fig. 1(b), and is obtained as follows:

$Q_{\text {all }} / V=I$

$V w / I=\rho_{\mathrm{r}}$

where $Q_{\mathrm{all}}[\mathrm{W} / \mathrm{m}], V[\mathrm{~V}], I[\mathrm{~A} / \mathrm{m}]$, and $w[\mathrm{~m}]$ are the total Joule heat per unit length, the electric potential difference between two REBCO layers, the current per unit length $(1.0 \mathrm{~A} / \mathrm{cm}$ constant in this paper), the tape width (4.0 $\mathrm{mm}$ in this paper), respectively.

Fig. 4 shows the coil-radial contact resistivity $\rho_{\mathrm{r}}$ vs. the turn-to-turn contact surface resistivity $\rho_{\mathrm{ct}}$. From Fig. 4, when $\rho_{\mathrm{ct}}<10^{-3}, \rho_{\mathrm{r}}$ is constant, i.e., $\rho_{\mathrm{r}}$ is dominated by the copper stabilizer. On the other hand, when $\rho_{\mathrm{ct}}>1, \rho_{\mathrm{r}}$ is linearly proportional to $\rho_{\mathrm{ct}}$, i.e., $\rho_{\mathrm{r}}$ is dominated by the turn-to-turn contact surface. Therefore, when the contact resistance is enough high, such as $70 \mu \Omega \cdot \mathrm{cm}^{2}$ [3], the coil-radial contact resistivity can be used as the turn-to-turn contact surface resistivity.

\section{Joule Heat Loss}

Next, we investigate the Joule heat loss per unit length. Fig. 5 shows the heat density per unit length of every component and the contact surface, where $Q_{\mathrm{Cu}, \mathrm{l}}, Q_{\mathrm{Cu}, \mathrm{r}}, Q_{\mathrm{Has}, \mathrm{l}}, Q_{\mathrm{ct}}$, and $Q_{\text {all }}$ are the heat density of the copper stabilizer of the left and right REBCO tape, the Hastelloy substrate of the left REBCO tape, the contact surface, and the total, respectively. When $\rho_{\mathrm{ct}}<10^{-1}$, $Q_{\text {all }}$ is almost constant, and is mainly composed of $Q_{\mathrm{Cu}, \mathrm{l}}$ and

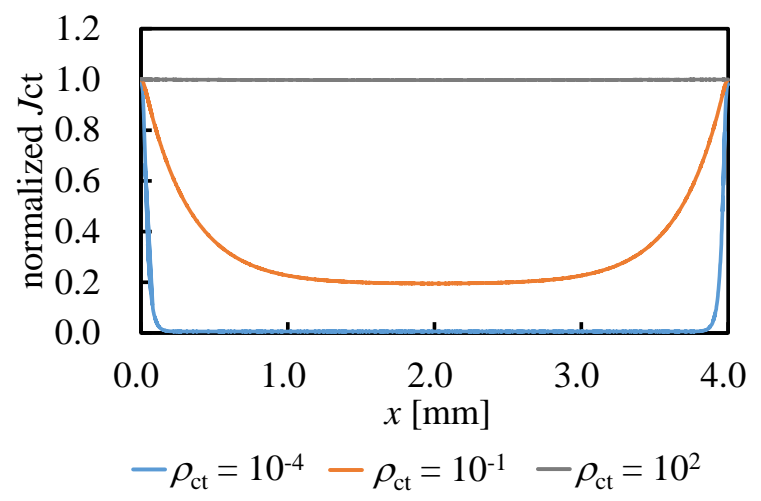

Fig. 3. Normalized current density passing through the turn-to-turn contact surface as a function of the position of contact surface $x$.

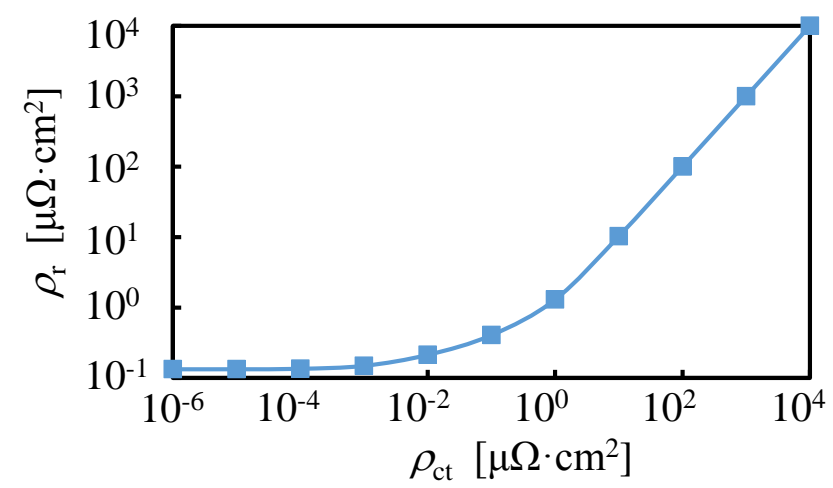

Fig. 4. Coil-radial contact resistivity $\rho_{\mathrm{r}}$ vs. turn-to-turn contact surface resistivity $\rho_{\mathrm{ct}}$.

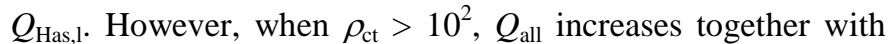
$Q_{\mathrm{ct}}$. When the turn-to-turn contact resistivity $\rho_{\mathrm{ct}}$ is high, $Q_{\text {Has, } 1}$ becomes larger than $Q_{\mathrm{Cu}, 1}$.

\section{Simple Equivalent Circuit}

Finally, we construct a simple equivalent circuit of the turnto-turn contact between the REBCO layers, and it represents the coil-radial contact resistance. Fig. 6 shows the proposed equivalent turn-to-turn contact circuit consisting of the resistance of the left and right copper stabilizers, $R_{\mathrm{Cu}, \mathrm{l}}$ and $R_{\mathrm{Cu}, \mathrm{r}}$, the Hastelloy substrate, $R_{\text {Has, }}$, and the contact surface, $R_{\mathrm{ct}}$. The copper stabilizer resistance is as a function of the turn-to-turn contact surface resistivity, meanwhile the Hastelloy substrate and contact surface resistances are constant. Fig. 7 shows the resistances per unit length, $R_{\mathrm{Cu}, \mathrm{l}}, R_{\mathrm{Cu}, \mathrm{r}}, R_{\mathrm{Has}, \mathrm{l}}$, and $R_{\mathrm{ct}}$.

The left copper stabilizer resistance $R_{\mathrm{Cu}, \mathrm{l}}$ changes depending on the length of current flow in the copper. $R_{\mathrm{Cu}, \mathrm{l}}$ becomes large when the current flows along the contact surface, as shown in Fig. 2(b). On the other hand, although the current also flows along the contact surface at $\rho_{\mathrm{ct}}=10^{2} \mu \Omega \cdot \mathrm{cm}^{2}$, as shown in Fig. 2(c), $R_{\mathrm{Cu}, \mathrm{l}}$ is small owing to a large amount of current following in the Hastelloy substrate. The current of the copper stabilizer on the left turn per unit length of longitudinal direction $I_{\mathrm{Cu}, \mathrm{l}}$ is obtained from

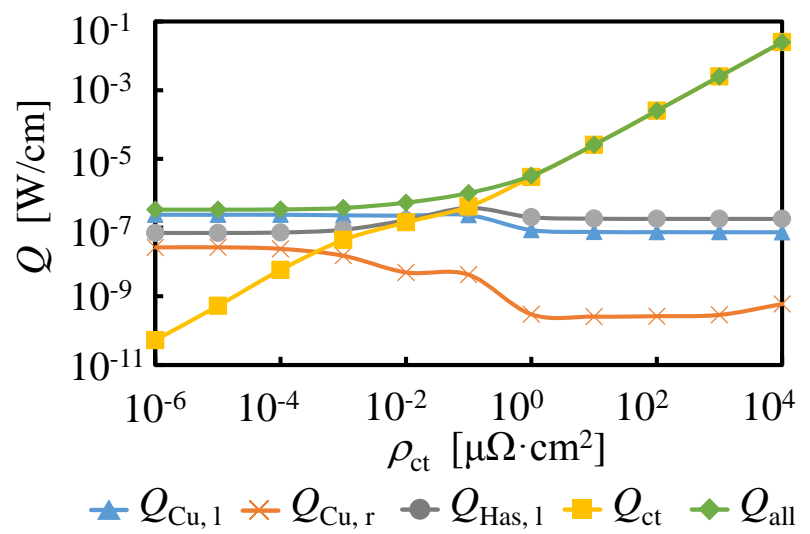

Fig. 5. Heat density of every material as a function of the turn-to-turn contact resistivity $\rho_{\mathrm{ct}} . Q_{\mathrm{Cu}, \mathrm{l}}, Q_{\mathrm{Cu}, \mathrm{r}}, Q_{\mathrm{Has}, \mathrm{l}}, Q_{\mathrm{ct}}$, and $Q_{\mathrm{all}}$ are the heat density of the copper stabilizer of the left and right REBCO tapes, the Hastelloy substrate of the left REBCO tape, the turn-to-turn contact, and the total, respectively.

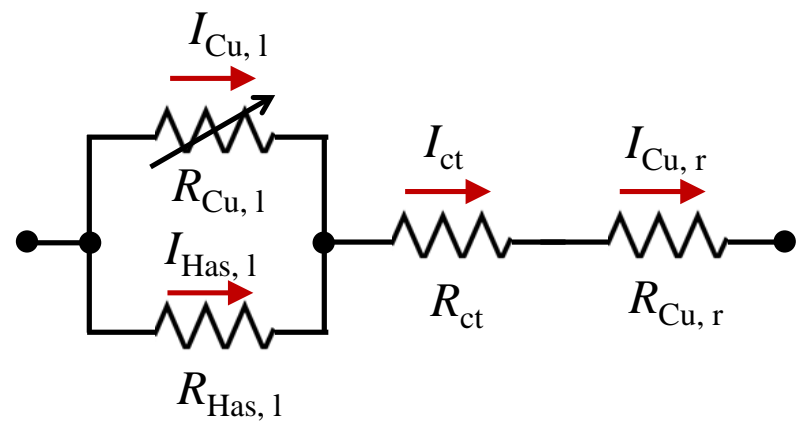

Fig. 6. Simple equivalent turn-to-turn contact circuit consisting of the resistance of the left and right copper stabilizers, the Hastelloy substrate, and the contact surface. $I_{\mathrm{Cu}, 1}$ and $I_{\mathrm{Has}}$, , are the current of copper stabilizer and Hastelloy substrate on the left turn, $I_{\mathrm{ct}}$ is the current through the contact surface, and $I_{\mathrm{Cu}}$, is the current of the copper stabilizer on the right turn, respectively. 


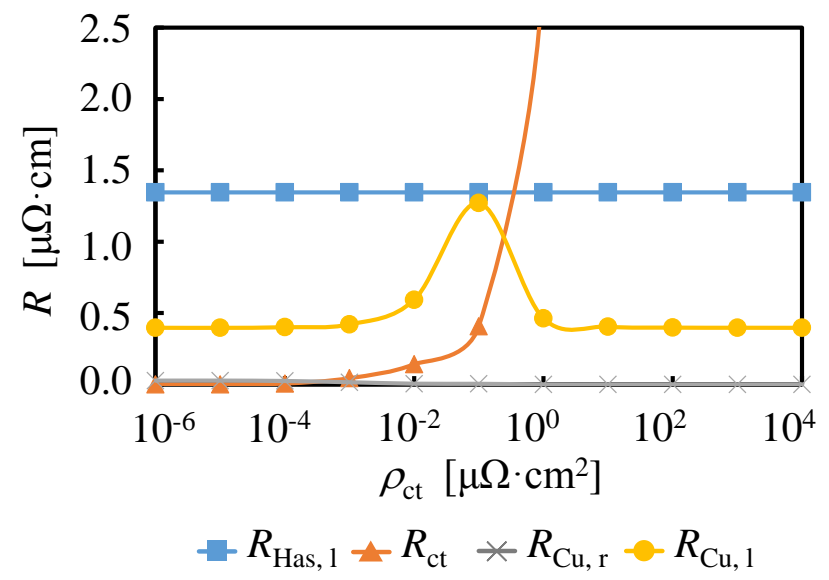

Fig. 7. Resistance per unit length of the left and right copper stabilizers, $R_{\mathrm{Cu}, \mathrm{I}}$ and $R_{\mathrm{Cu}, \mathrm{r}}$, the Hastelloy substrate, $R_{\mathrm{Has}, \mathrm{l}}$, and the contact surface, $R_{\mathrm{ct}}$.

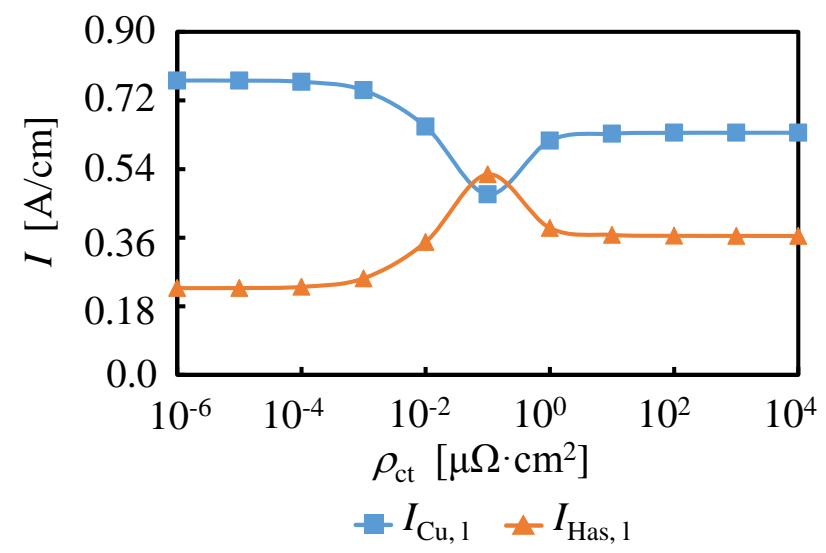

Fig. 8. Current per unit length of the left copper stabilizer and the Hastelloy substrate as a function of the contact surface resistivity $\rho_{\mathrm{ct}}$.

$I_{\mathrm{Cu}, \mathrm{l}}=I \frac{Q_{\mathrm{Cu}, 1}}{Q_{\mathrm{Cu}, 1}+Q_{\mathrm{Has}, 1}}$.

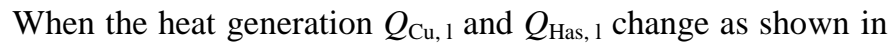
Fig. 5, the currents $I_{\mathrm{Cu}, \mathrm{l}}$ and $I_{\mathrm{Has,}}$ also change with the change of the contact resistivity. Fig. 8 shows the current of the left copper stabilizer and Hastelloy substrate, and Fig. 9 shows the current distribution on the half top of the REBCO tape at $\rho_{\mathrm{ct}}=$ $10^{-4}, 10^{-1}$, and $10^{2} \mu \Omega \cdot \mathrm{cm}^{2}$, where the color scale is different from that in Fig. 2 to focus on the current in the Hastelloy substrate. From Figs. 8 and 9, the current in the Hastelloy is relatively large at the large contact surface resistivity.

Next, we obtain the ideal resistance value of the Hastelloy substrate and the right copper stabilizer. The ideal Hastelloy substrate resistance $R_{\mathrm{Has}, \mathrm{l}}$ is $1.58 \mu \Omega \cdot \mathrm{cm}(=1.25 \mu \Omega \cdot \mathrm{m} \times 50$ $\mu \mathrm{m} / 3.96 \mathrm{~mm}$ ), and the ideal right copper stabilizer $R_{\mathrm{Cu}, \mathrm{r}} 1.00$ $\mathrm{n} \Omega \cdot \mathrm{cm}(=2.00 \mathrm{n} \Omega \cdot \mathrm{m} \times 20 \mu \mathrm{m} / 4 \mathrm{~mm})$. When $\rho_{\mathrm{ct}}>10^{2}$, it is assumed that most of the current of the left copper stabilizer flows directly into the right REBCO tape. Therefore, the ideal $R_{\mathrm{cu}, \mathrm{l}}$ is $0.35 \mu \Omega \cdot \mathrm{cm}(=2.00 \mathrm{n} \Omega \cdot \mathrm{m} \times 70 \mu \mathrm{m} / 40 \mu \mathrm{m})$. These ideal values roughly agree with the values in Fig. 8. As a result, although the turn-to-turn contact surface resistance is a

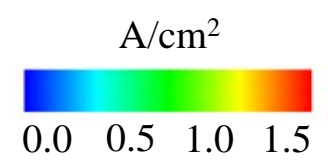

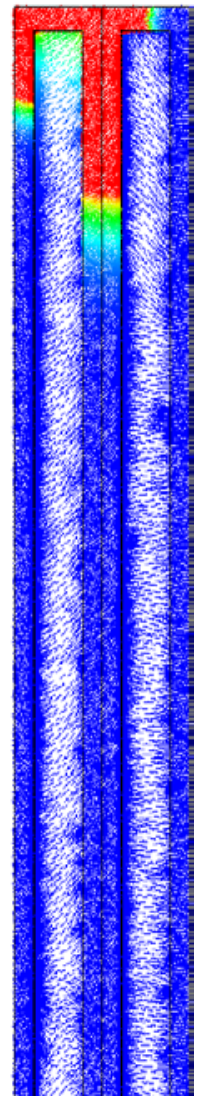

(a)

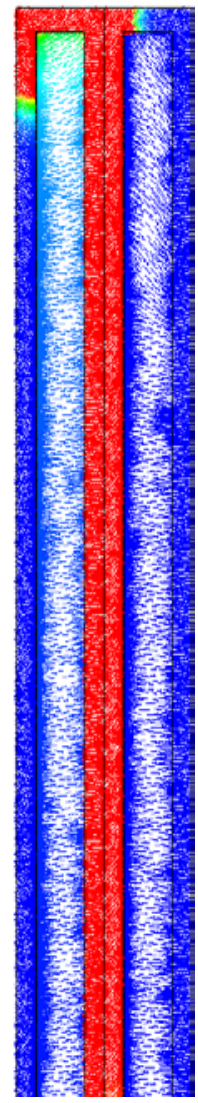

(b)

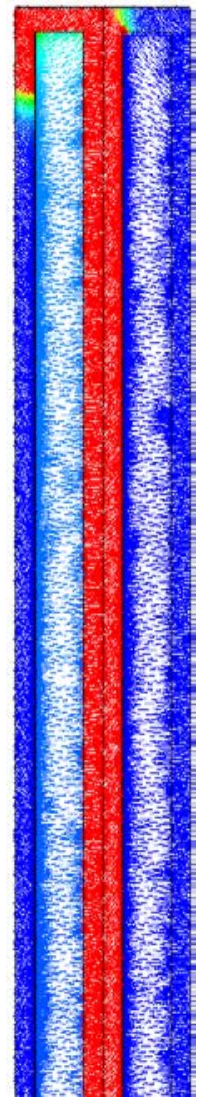

(c)
Fig. 9. Current distribution in the Hastelloy substrate at (a) $\rho_{\mathrm{ct}}=10^{-4}$, (b) $10^{-1}$, and (c) $10^{2} \mu \Omega \cdot \mathrm{cm}^{2}$, respectively.

major contributing factor, we can simply estimate the current behavior using the proposed simple equivalent circuit with the ideal resistance. It is very useful in the determination of the parameters of REBCO tape, such as copper stabilizer thickness, and it is possible to roughly estimate the Joule heat.

\section{CONCLUSION}

The NI winding technique for REBCO pancake is expected to be applied to NMR, MRI and accelerator applications to enhance the thermal stability and the current density. Therefore, it is necessary to investigate the cross-sectional structure of the REBCO tape, suitable for the NI winding technique. In this paper, we showed the bypass current simulation method to clarify the current behavior and the Joule heat loss, and proposed the simple equivalent circuit for the turn-to-turn contact. Through these results, it is possible to investigate the cross-sectional structure of the REBCO tape, e.g., the copper stabilizer thickness and the tape width.

In the future study, we have to simulate the bypass current behavior on the cross section of more than two REBCO tapes. 


\section{REFERENCES}

[1] S. Hahn, D. K. Park, J. Bascuñán, and Y. Iwasa, "HTS pancake coils without turn-to-turn insulation,” IEEE Trans. Appl. Supercond., vol. 21, no. 3, pp. 1592-1595, Jun. 2011.

[2] S. B. Kim et al., "The characteristics of the normal-zone propagation of the HTS coils with inserted Cu tape instead of electrical insulation," IEEE Trans. Appl. Supercond., vol. 22, no. 3, Jun. 2012, Art. ID. 4701504.

[3] Y. Iwasa, J. Bascuñán, S. Hahn, J. Voccio, Y. Kim, T. Lécrevisse, J. Song, and K. Kajikawa, "A high-resolution 1.3-GHz/54-mm LTS/HTS NMR magnet,” IEEE Trans. Appl. Supercond., vol. 25, no. 3, Jun. 2015, Art. ID. 4301205.

[4] X. Wang, T. Wang, E. Nakada, A. Ishiyama, R. Itoh, and S. Noguchi, “Charging Behavior in No-Insulation REBCO Pancake Coils," IEEE Trans. Appl. Supercond., vol. 25, no. 3, Jun. 2015, Art. ID. 4601805.

[5] Y. Kim et al., "Numerical analysis on bifurcated current flow in noinsulation magnet," IEEE Trans. Appl. Supercond., vol. 24, no. 3, Jun. 2014, Art. ID. 4900404.

[6] T. Wang, S. Noguchi, X. Wang, I. Arakawa, K. Minami, K. Monma, A. Ishiyama, S. Hahn, and Y. Iwasa, "Analyses of transient behaviors of no-insulation REBCO pancake coils during sudden discharging and over current,” IEEE Trans. Appl. Supercond., vol. 25, no. 3, Jun. 2015, Art. ID. 4603409.

[7] K. L. Kim, S. Hahn, Y. Kim, D. G. Yang, J. Song, J. Bascuñán, H. Lee, and Y. Iwasa, "Effect of winding tension on electrical behaviors of a noinsulation ReBCO pancake coil,” IEEE Trans. Appl. Supercond., vol. 24, no. 3, Jun. 2014, Art. ID. 4600605.

[8] X. Wang et al., "Turn-to-turn contact characteristics for an equivalent circuit model of no-insulation ReBCO pancake coil," Supercond. Sci. Technol., vol. 26, no. 3, Mar. 2013, Art. ID. 035012.

[9] http://www.superpower-inc.com/content/2g-hts-wire

[10] Y. Kamiya and T. Onuki, "3D eddy current analysis by the finite element method using double nodes technique,” IEEE Trans. Magn., vol. 32, no. 3, pp. 741-744, May 1996. 